\begin{abstract}
Resumen
La anticoncepción de emergencia se refiere al uso de medicación o a la inserción de un dispositivo intrauterino de cobre para prevenir el embarazo luego de una relación sexual no protegida o del fallo de un método anticonceptivo, en una mujer que no desea el embarazo. De entre las alternativas farmacológicas del método, se estima que el uso de levonorgestrel prevendría el $50 \%$ de los embarazos.

A partir de una viñeta clínica, en la cual se describe el caso de una paciente obesa que resultó embarazada luego de utilizar anticoncepción hormonal de emergencia tras una relación sexual no protegida, se realizó una búsqueda bibliográfica para establecer la efectividad del método en pacientes con esta característica (obesidad). La búsqueda identificó un metanálisis que relacionó al triple la probabilidad de embarazo en pacientes con índice de masa corporal superior a $30 \mathrm{~kg} / \mathrm{m} 2$ que utilizaron anticoncepción de emergencia hormonal.
\end{abstract}

\title{
Abstract
}

Emergency contraception refers to the use of medication or the insertion of a copper intrauterine device to prevent pregnancy after the occurance of unprotected sex intercourse or failure of a contraceptive method in a woman who does not desire pregnancy. Among the pharmacological alternatives of the method it is estimated that the use of levonorgestrel prevents $50 \%$ of pregnancies.

From a clinical vignette, in which the case of an obese patient who became pregnant using hormonal emergency contraception after unprotected sex intercourse is described, a literature search was conducted to establish the effectiveness of the method in patients with this feature (obesity). The search identified a meta-analysis that shows an incresed probability (three times) of pregnancy in patients with body mass index greater than $30 \mathrm{~kg} / \mathrm{m} 2$ that used hormonal emergency contraception.

Teste M. La anticoncepción de emergencia es menos efectiva en mujeres con obesidad. Evid Act Pract Ambul. 2016 ;19(3):97-98.

\section{Viñeta Clínica}

Se presenta a la consulta una paciente de 26 años de edad, con antecedente de obesidad, que consulta por retraso menstrual. Refiere haber presentado una relación no protegida hace un mes, luego de la cual accedió a la anticoncepción de emergencia con levonorgestrel, utilizada dentro de las $72 \mathrm{hs.} \mathrm{Luego}$ del consumo de la misma presentó metrorragia escasa y desde entonces no se reanudó su ciclo menstrual. Durante la consulta se realiza un test de embarazo que resulta positivo.

\section{Pregunta que generó el caso}

¿La anticoncepción de emergencia tiene la misma eficacia en pacientes obesas que en pacientes no obesas?

\section{Estrategia de búsqueda}

Se realizó una búsqueda en MEDLINE usando la combinación de los términos ("Contraception, Postcoital"[Mesh]) AND "Obesity"[Mesh] y términos ("Contraception, Postcoital"[Mesh]) AND obese. Se obtuvieron cuatro artículos.

\section{Resultados de la búsqueda}

1. Batur P. Emergency contraception: Separating fact from fiction. Cleveland Clinic Journal of Medicine 2012 Nov; 79(11):771-776. doi:10.3949/ccjm.79a.12019. ${ }^{1}$

2. White K. Update on contraception. Seminars in Reproductive Medicine. 2010 Mar; 28(2):93-4. doi: 10.1055/s-0030-1248132. Epub 2010 Mar 29. ${ }^{2}$

3. Glasier A y col. Can we identify women at risk of pregnancy despite using emergency contraception? Data from randomized trials of ulipristal acetate and levonorgestrel. Contraception 2011 Oct; 84(4):363-7. doi: 10.1016/j.contraception.2011.02.009. Epub 2011 Apr 2. ${ }^{3}$

4. Lalitkumar $P$ y col. Emergency contraception. Best Pract Res ClinEndocrinolMetab. $2013 \quad$ Feb;27(1):91-101. doi: 10.1016/j.beem.2012.09.003. Epub 2012 Oct $11 .^{4}$

Dado que todos los artículos hacían referencia al estudio descripto en el artículo "Can we identify women at risk of pregnancy despite using emergency contraception?", hemos decidido seleccionarlo como única evidencia.
Algunos aspectos sobre anticoncepción de emergencia La anticoncepción de emergencia se refiere al uso de medicación o a la inserción de un dispositivo intrauterino de cobre para prevenir el embarazo luego de una relación sexual no protegida o del fallo de un método anticonceptivo, en una mujer que no desea el embarazo.

En Argentina contamos con el levonorgestrel consumido en una dosis de $1,5 \mathrm{mg}$ dentro de las $72 \mathrm{hs}$ de embarazo o bien con el Método Yuzpe, que utiliza anticonceptivos orales combinados a altas dosis. Luego de la promulgación de la Ley Nacional № 25.673/02 a través de la cual se creó el Programa Nacion de Salud Sexual y Procreación Responsable, entre otras cosas, se establece la responsabilidad del Estado de garantizar el derecho de toda persona a disponer y elegir el método anticonceptivo de preferencia dentro de los cuales se encuentra la anticoncepción hormonal de emergencia.

Se estima que la anticoncepción de emergencia con levonorgestrel prevendría hasta un $50 \%$ de los embarazos.

Actualmente en otro países se dispone de otros métodos, como la inserción del dispositivo intrauterino dentro de las 120 hs de producida la relación no protegida y el consumo oral del acetato de ulipristal dentro de las $120 \mathrm{hs}$.

\section{Resumen de la evidencia}

En orden de explorar posible factores que expliquen el aumento del riesgo de fallas de los anticonceptivos de emergencia, se utilizó en el artículo seleccionado, la información de un metaanálisis que había evaluado dos estudios aleatorizados y controlados que habían comparado la eficacia del ulipristal con el levonorgestrel.

Ambos ensayos clínicos tuvieron un diseño similar. Uno ${ }^{5}$ había reclutado mujeres que habían tenido una relación sexual no protegida durante las 72 horas previas y el otro, mujeres que la habían tenido hasta 120 horas antes ${ }^{6}$. Ambos estudios incluyeron mujeres fértiles con ciclos regulares que no venían usando anticoncepción hormonal. Excluyeron a las que habían sido esterilizadas (ellas o sus parejas), venían usando dispositivo intrauterino (DIU), estaban en período de lactancia y/o tenían menos de 16 años.

El tratamiento fue administrado luego de excluir el embarazo a través de un análisis de orina. Para evaluar el resultado, fue

* Servicio de Medicina Familiar y Comunitaria del Hospital Italiano de Buenos Aires maryteste@ gmail.com 
realizada una prueba de embarazo una semana después del inicio esperado de la menstruación.

Se buscó el efecto se las siguientes covariables: edad, índice de masa corporal (IMC), peso, horas desde la relación no protegida hasta el consumo de la anticoncepción de emergencia, ocurrencia de otras relaciones no protegidas luego del consumo de la anticoncepción de emergencia, antecedente de embarazo y probabilidad de concepción definida por el método Trussell et $\mathrm{al}^{7}$.

De las covariables analizadas, tres mostraron tener efecto estadísticamente significativo en el riesgo de embarazo: índice de masa corporal (IMC), probabilidad de concepción y presencia de otras relaciones luego del consumo de anticoncepción de emergencia. La de mayor impacto fue el IMC.

Un IMC superior a $30 \mathrm{~kg} / \mathrm{m}^{2}$ se asoció al triple de probabilidad de concepción (OR 3,60; IC95\% 1,96 a 653; p < 0,0001), con un efecto más notorio para el levonorgestrel $(4,41 ; 2,05$ a 9,44 ; $\mathrm{p}=0,0002$ ).

\section{Conclusión}

Teniendo en cuenta los resultados del meta-análisis que hemos resumido, podemos concluir que hay una importante disminución del efecto anticonceptivo de emergencia del levonorgestrel en las pacientes con obesidad.

De todas formas, hay que tener en cuenta que ninguno de los estudios utilizados fue diseñado específicamente para evaluar dicho efecto. A su vez, el número total de embarazos ocurridos fue pequeño $(n=60)$ y menor aún en el subgrupo de mujeres obesas $(n=20)$ y/o que usaron levonorgestrel $(n=14)$.

Es por esto que es difícil distinguir si dicho efecto fue únicamente por la obesidad o si entran en juego otros confundidores. Por otro lado, y si bien la eficacia de la anticoncepción de emergencia podría disminuir significativamente según este meta análisis, su efecto no es nulo. Por lo tanto, y teniendo en cuenta que es el único método disponible hasta el momento en la Argentina, consideramos que se debe seguir siendo recomendado su uso cuando este indicado, mientras esperamos el desarrollo de otros métodos que podrían ser más eficaces.

\section{Referencias:}

1. Batur P. Emergency contraception: Separating fact from fiction. Cleveland Clinic Journal of Medicine 2012 Nov; 79(11):771-776. doi:10.3949/ccim.79a.12019. Erratum in: Cleve Clin J Med. 2013 Aug;80 (8):482. Dosage error in article text.

2. White K. Update on contraception. Seminars in Reproductive Medicine. 2010 Mar; 28(2):93-4. doi: 10.1055/s-0030-1248132. Epub 2010 Mar 29.

3. Glasier A col. Can we identify women at risk of pregnancy despite using emergency contraception? Data from randomized trials of ulipristal acetate and levonorgestrel.Contraception 2011 Oct; 84(4):363-7. doi: 10.1016/j.contraception.2011.02.009. Epub 2011 Apr 2

4. Lalitkumar P y col. Emergency contraception. Best Pract Res ClinEndocrinolMetab. 2013 Feb;27(1):91-101. doi: 10.1016/.beem.2012.09.003. Epub 2012 Oct 1.

5. Creinin M y col. Progesterone receptor modulator for emergency contraception: a randomized controlled trial. ObstetGynecol 2006;108:1089-97.

6. Glasier A y col. Ulipristal acetate versus levonorgestrel for emergency contraception: a randomized non-inferiority trial and meta-analysis. Lancet 2010; 375:555-62

7.Trussell J y col. New estimates of theeffectiveness of theYuzperegimen of emergencycontraception. Contraception 1998; 57:363-9.

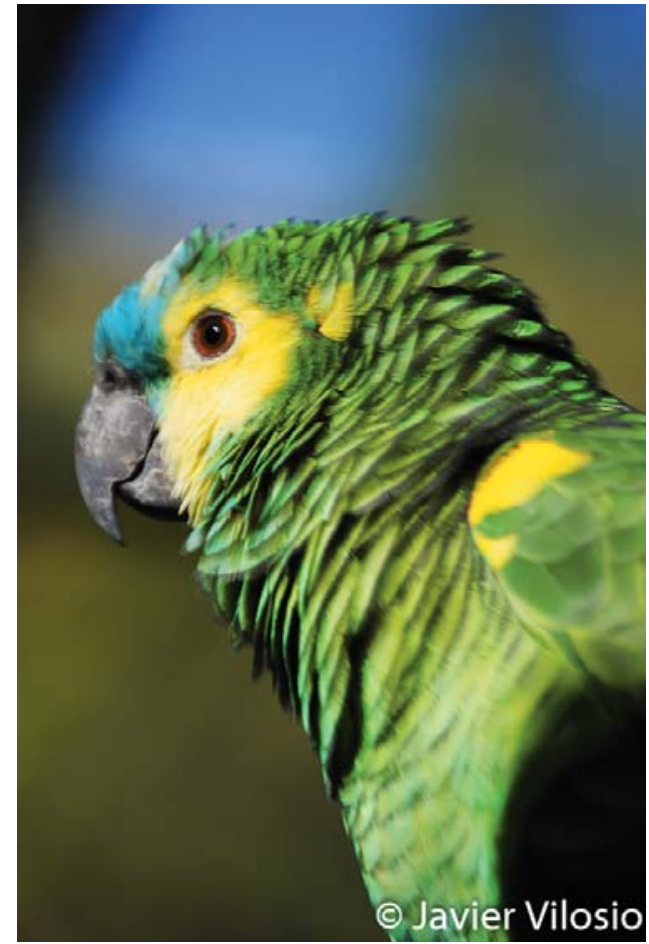

Cortesía: Javier Vilosio

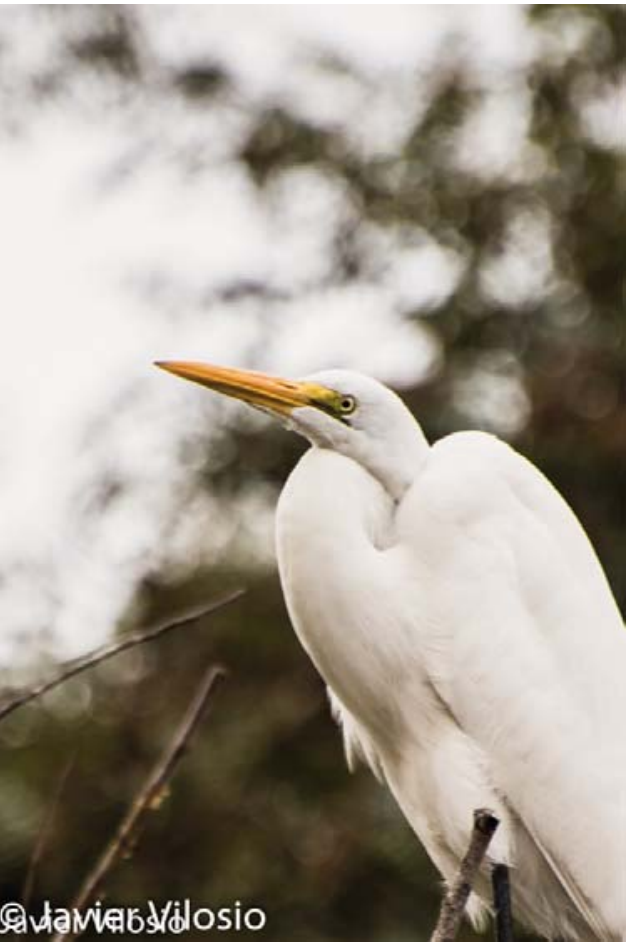

